ELECTRONIC RESEARCH ANNOUNCEMENTS OF THE AMERICAN MATHEMATICAL SOCIETY

Volume 12, Pages 19-28 (March 2, 2006)

S $1079-6762(06) 00156-9$

\title{
VANISHING OF THE ENTROPY PSEUDONORM FOR CERTAIN INTEGRABLE SYSTEMS
}

\author{
BORIS S. KRUGLIKOV AND VLADIMIR S. MATVEEV
}

(Communicated by Boris Hasselblatt)

\begin{abstract}
We introduce the notion of entropy pseudonorm for an action of $\mathbb{R}^{n}$ and prove that it vanishes for the group actions associated with a large class of integrable Hamiltonian systems.
\end{abstract}

\section{ENTROPY PSEUDONORM}

Let $W$ be a smooth manifold and $\Phi:\left(\mathbb{R}^{n},+\right) \rightarrow \operatorname{Diff}(W)$ a smooth action on it. Assume there exists a compact $\Phi$-invariant exhaustion of $W$, i.e., $W=\bigcup_{i=1}^{\infty} K_{i}$ with compact $\Phi$-invariant sets $K_{i}$. Define the following function on $\mathbb{R}^{n}$ (where $h_{\text {top }}$ is the topological entropy):

$$
\rho_{\Phi}(v)=h_{\mathrm{top}}(\Phi(v)), \quad v \in \mathbb{R}^{n} .
$$

This function is a pseudonorm on $\mathbb{R}^{n}: \rho_{\Phi}(v)$ is well defined because, under our hypothesis, the entropy $h_{d}$ of $[8$ does not depend on the distance function $d$ on $W$, homogeneity is standard, and the triangle inequality follows from the $\mathrm{Hu}$ formula [16. We call $\rho_{\Phi}$ the entropy pseudonorm.

We will investigate it in the case of the Poisson action corresponding to an integrable Hamiltonian system on a symplectic manifold $\left(W^{2 n}, \omega\right)$. Namely, let $\left(W^{2 n}, \omega\right)$ possess pairwise Poisson-commuting functions $I_{1}, I_{2}, \ldots, I_{n}$, which are functionally independent almost everywhere.

Denote by $\varphi_{i}^{\tau}$ the time $\tau$ shift along the Hamiltonian vector field of the function $I_{i}$. The maps $\varphi_{i}^{\tau}$ commute and therefore generate the Poisson action of the group $\left(\mathbb{R}^{n},+\right)$,

$$
\Phi\left(\tau_{1}, \ldots, \tau_{n}\right) \stackrel{\text { def }}{=} \varphi_{1}^{\tau_{1}} \circ \cdots \circ \varphi_{n}^{\tau_{n}}: W^{2 n} \rightarrow W^{2 n},
$$

with the corresponding momentum map $\Psi=\left(I_{1}, \ldots, I_{n}\right): W^{2 n} \rightarrow \mathbb{R}^{n}$; see [1].

The entropy pseudonorm $\rho_{\Phi}$ vanishes in the following important cases (we discuss these conditions at the end of the paper, in Section 31):

- Williamson-Vey-Eliasson-Ito nondegenerate singularities [12, 18;

- Taimanov nondegeneracy condition 33.

In the first case vanishing of topological entropy of the Hamiltonian flow was proved in [29]; in the second case, in [33]. Since there is nothing special about

Received by the editors October 4, 2005 and, in revised form, November 29, 2005.

2000 Mathematics Subject Classification. Primary 37C85, 37J35, 37B40; Secondary 70H07, $37 \mathrm{~A} 35$.

(C)2006 American Mathematical Society Reverts to public domain 28 years from publication 
the Hamiltonian in these situations, it can be changed to any of the integrals and $\rho_{\Phi} \equiv 0$ follows. Also in [28, 9] vanishing of $h_{\text {top }}$ was proven for the cases of

- Systems integrable with periodic integrals;

- Collectively integrable systems (the definition is in [14]).

It is not difficult to see that in both cases the entropy pseudonorm $\rho_{\Phi}$ vanishes as well.

Note that Liouville integrability does not imply vanishing of topological entropy; see 7] (more examples in [10]). For these examples the entropy pseudonorm is nonzero, but degenerate. However, it is possible to construct integrable examples [20] such that $\rho_{\Phi}$ is a norm.

In the present paper we prove vanishing of the entropy pseudonorm for another class of integrable systems. These systems have recently been actively studied in mathematical physics in the framework of the theory of separation of variables. In different contexts they are called Benenti systems [17, L-systems [3, 4, cofactor systems [23, or quasi-bi-Hamiltonian systems [11]. The Benenti systems are certain integrable Hamiltonian systems on $T^{*} M$ with Hamiltonian of the form $H=K_{g}+V$, where $K_{g}: T^{*} M \rightarrow \mathbb{R}, K_{g}(x, p)=\langle p, p\rangle_{g}$, is twice the kinetic energy corresponding to a Riemannian metric $g$ and $V: M \rightarrow \mathbb{R}$ is a potential. Important feature of these systems is that every integral is a sum of a function quadratic in momenta and a function on $M$. Moreover, the quadratic forms corresponding to the quadratic-in-momenta terms are simultaneously diagonalizable. We will provide precise definitions, the conditions on the metric $g$ and potential $V$ as well as formulas for integrals in Section 2.1, where we also explain how these systems are related to the theory of geodesic equivalence.

Theorem 1. Let $M$ be a compact connected manifold. Then the entropy pseudonorm of the action $\Phi$ associated with any Benenti integrable Hamiltonian system on $T^{*} M$ vanishes: $\rho_{\Phi} \equiv 0$.

The class of mechanical systems covered by Theorem 1 contains Lagrange spinning tops, the von Neumann system, the Braden system, Bogoyavlensky systems, some Manakov systems, and many other quadratically integrable (Stäckel) systems. For most of them our vanishing result is new.

For geodesic flows $(V \equiv 0)$ vanishing of $h_{\text {top }}(H)$ was proven in our earlier paper 21. Theorem 1 generalizes the result of [21] in the following two directions. First, it includes the potential energy in the picture. Second, it shows that the topological entropy of the Hamiltonian flow of every integral (not only the Hamiltonian) vanishes.

Our result has topological implications (for example, due to 31]). We discussed some of them in 21] (see also [25, 26, 27]). In particular, if a manifold $M$ possesses a Benenti-integrable Hamiltonian system, then it must be covered by a product of a rationally elliptic manifold and a torus: $M^{n}=Q^{k} \tilde{\times} T^{n-k}$. Indeed, by the description from Section 2.1 we know that the kinetic energy $K_{g}$ corresponding to a Benenti system is also an integrable Hamiltonian and, moreover, for the corresponding Riemannian metric $g$, there exists another, $\bar{g}$, which is geodesically equivalent to $g$ and is strictly nonproportional to it. Thus the claim follows from Theorem 7 of [21].

There are also many dynamical implications. Actually, for large values of the Hamiltonian $H$ and the integrals $I_{t}$ (with $t \leq \min \operatorname{Sp}(L)$ to achieve positivity of the 
quadratic forms; see Section 2.1), these functions behave like Riemannian metrics on $M$ because of the Maupertuis principle [1, 5]. Their geodesic flows commute and have zero topological entropy. This implies, for all of them, a subexponential growth of the volume of balls in the universal cover (Manning's inequality), of the number of geodesic arcs joining two generic points (Mañé's formula), etc.; see 30] (Sections 3.4, 3.5, and 4.2).

An outline of the proof for Theorem 1 will be presented in Section 2.2. We describe singular orbits of the Poisson action and show that the restriction of our integrable system to every singular orbit is a subsystem of a Benenti system on a manifold of smaller dimension. Then we apply induction on the dimension. Again as in the case of geodesic flows, the set of singular points can be very complicated: If $n \stackrel{\text { def }}{=} \operatorname{dim}(M)>2$, then there exists a singular point over every point of $M$ and the set of singular points in $\Psi^{-1}(c)$ can project to a fractal in $M^{n}$ of Hausdorff dimension $>n-1$.

We also discuss vanishing of other entropies. The well-known entropy for the group action $h_{\text {top }}(\Phi)$ [13] vanishes for Benenti systems by elementary reasons: $h_{\text {top }}(\Phi)=0$. Actually, if this entropy is positive, then (directly from the definition) all entropies of subgroup actions are infinite; in particular, $h_{\text {top }}(H)=+\infty$, which is incorrect.

However, there is another definition of the entropy for a group action $h_{U}(\Phi)$, which behaves naturally with respect to restrictions to subgroup actions [15. Here $U$ is the cube $[-1,1]^{n} \subset \mathbb{R}^{n}$ that defines the strongly regular system $(U, 2 U, 3 U, \ldots)$ exhausting our group $\left(\mathbb{R}^{n},+\right)$. With respect to this definition and the action $\Phi$ of $\mathbb{R}^{n}$ associated to a Benenti integrable system we have (see [15] for the definition and properties)

$$
h_{U}(\Phi)=0 .
$$

This follows from the inequalities

$$
\max _{1 \leq k \leq n} h_{\text {top }}\left(I_{k}\right) \leq \sup _{v \in U} \rho_{\Phi}(v) \leq h_{U}(\Phi) \leq n \cdot \max _{1 \leq k \leq n} h_{\text {top }}\left(I_{k}\right) .
$$

Here the first inequality is obvious, the second is Proposition 2.6 from 15, and the third easily follows from the definition of $h_{U}$. Thus vanishing of the entropy pseudonorm $\rho_{\Phi}$ is equivalent to vanishing of the entropy $h_{U}(\Phi)$.

\section{Definitions And SKetch of the PRoOF}

2.1. Benenti systems and geodesically equivalent metrics. Let $g, \bar{g}$ be two Riemannian metrics on a connected manifold $M$ of dimension $n$, and $b_{g}: T M \rightarrow$ $T^{*} M, \sharp^{\bar{g}}: T^{*} M \rightarrow T M$ the corresponding bundle morphisms. We regard the bundle morphism $\sharp \bar{g} \circ b_{g}: T M \rightarrow T M$ as a $(1,1)$-tensor.

The metrics $g, \bar{g}$ are called geodesically equivalent if every geodesic of $\bar{g}$, regarded as an unparameterized curve, is a geodesic of $g$. They are said to be strictly nonproportional at $P \in M^{n}$ if the spectrum $\operatorname{Sp}\left(\sharp^{\bar{g}} \circ b_{g}\right) \subset \mathbb{R}_{+}$is simple at $P$.

Consider the $(1,1)$-tensor $L \stackrel{\text { def }}{=}\left(\sharp^{\bar{g}} \circ b_{g}\right) / \sqrt[n+1]{\operatorname{det}\left(\sharp^{\bar{g}} \circ b_{g}\right)}: T M \rightarrow T M$. For every $t \in \mathbb{R}$, consider the $(1,1)$-tensor $S_{t} \stackrel{\text { def }}{=} \operatorname{det}(L-t \mathrm{Id})(L-t \mathrm{Id})^{-1}$. The family $S_{t}$ is polynomial in $t$ of degree $n-1$. 
We will always identify the tangent and the cotangent bundle of $M$ with the help of $b_{g}$. This identification gives us a symplectic form and a Poisson structure on $T M$.

Theorem 2 ([24, 25]). If $g, \bar{g}$ are geodesically equivalent, then for all $t_{1}, t_{2} \in \mathbb{R}$ the functions

$$
I_{t_{i}}: T M \rightarrow \mathbb{R}, \quad I_{t_{i}}(v) \stackrel{\text { def }}{=} g\left(S_{t_{i}}(v), v\right)
$$

are commuting integrals for the geodesic flow of $g$.

If, in addition, the metrics are strictly nonproportional at one point, then this is so for almost every point. Consequently, for all $t_{1}<\cdots<t_{n}$ the integrals $I_{t_{i}}$ are functionally independent almost everywhere so that the geodesic flow of $g$ is Liouville-integrable.

It is possible to add potential energy to the picture. In local coordinates, it was done in [2] (see also [3, 4, 6]); other approaches are given in [17] and 11].

Let $g$ and $\bar{g}$ be geodesically equivalent Riemannian metrics on $M^{n}$. A smooth function $V: M^{n} \rightarrow \mathbb{R}$ will be called compatible with respect to $g$ and $\bar{g}$ if the 1 -form

$$
d V \circ(L-\operatorname{trace}(L) \mathrm{Id})
$$

is exact. For every pair of geodesically equivalent metrics that are not affine equivalent, we can prove the existence of a nonconstant compatible $V$ (actually of a continuum-dimensional family).

It is possible to show that if there exists a compatible function $V$, then there exists a family $V_{t}, t \in \mathbb{R}$, of smooth functions on $M^{n}$ such that the following two conditions are fulfilled:

$$
\left\{\begin{array}{l}
V_{t} \text { is a polynomial in } t \text { of degree } \leq n-1, \\
d V \circ S_{t}=d V_{t} \quad \text { for every } t \in \mathbb{R} .
\end{array}\right.
$$

The potential $V$ determines the family $V_{t}$ up to (addition of) a constant polynomial $P(t)$ of degree $\leq n-1$. Note that the family $V_{t}$ also defines the function $V$ up to a constant. In fact, the function $V$ is the coefficient of $t^{n-1}$.

Locally, the existence of such $V_{t}$ was explained in 4. From the normal form for the functions $V_{t}$, given in Theorem 4 below, it is clear that, near generic points, we have a great deal of freedom in choosing the functions $V$ and $V_{t}$ : They depend on arbitrary $n$ functions of one variable. Globally on $M$ the existence of such $V_{t}$ is nontrivial, for instance because the functions $X_{i}$ from Theorem 4 can have singularities near the bifurcation points of the spectrum $\operatorname{Sp}(L)$ of $L$.

Theorem 3 ([3, 4, 11, 6]). Let $g, \bar{g}$ on a connected $M^{n}$ be geodesically equivalent. Suppose $V$ is compatible with respect to $g, \bar{g}$. Consider a family $V_{t}$ of functions satisfying conditions (11). Then for all $t_{1}, t_{2} \in \mathbb{R}$ the functions $\hat{I}_{t_{i}} \stackrel{\text { def }}{=} I_{t_{i}}+V_{t_{i}}$ are commuting integrals for the Hamiltonian system with Hamiltonian $K_{g}+V$, where $K_{g}$ is twice the kinetic energy corresponding to $g$. If, in addition, the metrics are strictly nonproportional at least at one point, then for all $t_{1}<\cdots<t_{n}$ the integrals $\hat{I}_{t_{i}}$ are functionally independent almost everywhere.

We will call a Benenti system the integrable system on $T M^{n}$ generated by the integrals $\hat{I}_{t_{1}}, \ldots, \hat{I}_{t_{n}}$ from Theorem 3 provided the metrics $g, \bar{g}$ are strictly nonproportional at least at one (and hence at almost every) point. 
2.2. Idea of the proof of Theorem 1. We use induction on the dimension. If the dimension of the manifold is $n<2$, Theorem 1 is trivial. Assume that Theorem 1 is true for every dimension less than $n$ and consider $\operatorname{dim} M=n$.

Suppose the topological entropy of the Hamiltonian flow corresponding to an integral $\hat{I}_{t}$ is not zero. Then, by the variational principle, there exists an ergodic $\hat{I}_{t}$-invariant Borel probability measure $\mu$ such that $h_{\mu}(g) \neq 0$ [19]. By ergodicity the support $\operatorname{Supp}(\mu)$ is contained in a connected component of some fiber $\Psi^{-1}(c)$. If $\operatorname{Supp}(\mu)$ contains a point $P$ with $\operatorname{rank}\left(d \hat{I}_{t_{1}}, \ldots, d \hat{I}_{t_{n}}\right)=n$, then the orbit of $P$ is diffeomorphic to a cylinder over torus, $\Phi\left(\mathbb{R}^{n}, P\right) \simeq T^{k} \times \mathbb{R}^{n-k}, 0 \leq k \leq n$ [1]. By the implicit function theorem, a small neighborhood of a point $P$ in $\operatorname{Supp}(\mu)$ lies in the orbit $\Phi\left(\mathbb{R}^{n}, P\right)$. Since $\operatorname{Supp}(\mu)$ is a closed invariant subset and its point $P$ cannot be wandering, the support is diffeomorphic to a subtorus $T^{l}, l \leq k$, for which the flow of $\hat{I}_{t}$ is conjugate to a standard linear flow. This implies that the entropy $h_{\mu}$ vanishes.

Now suppose that every point of $\operatorname{Supp}(\mu)$ is singular, so that

$$
\operatorname{rank}\left(d \hat{I}_{t_{1}}, \ldots, d \hat{I}_{t_{n}}\right) \leq k<n
$$

and the rank equals $k$ almost everywhere on the support of $\mu$. In this case, we can reduce the dimension. Namely, there exists a closed proper submanifold $N^{k} \subset M^{n}$ with induced Benenti system and a subgroup $\mathbb{R}^{k} \subset \mathbb{R}^{n}$ with $\tilde{\Phi}=\Phi_{\mid \mathbb{R}^{k}}$ such that $\operatorname{Supp}(\mu)$ is $\tilde{\Phi}$-invariant and $\tilde{\Phi}_{\mid \operatorname{Supp}(\mu)}$ is a subsystem of the Poisson action corresponding to the Benenti system on $N^{k}$. Then $h_{\mu}=0$ by the induction assumption.

This is actually the main point of the proof. Precisely the same logic was used in [21. To a certain extent, not only the statement, but also most of the proofs from [21] can be generalized to our more general setting. In Sections 2.3, 2.4 we will explain how to construct these closed submanifolds under the additional assumption that all eigenvalues of $L$ are nonconstant. This additional assumption makes the proof much shorter (for instance, because in this case we can take $k=n-1$; in the paper 21] the longest part was dedicated to dealing with constant eigenvalues of $L)$, so that we can hope to make the main ideas of the proof clear to everyone.

\subsection{Benenti systems and singular points in Levi-Civita coordinates.}

Theorem 4 (follows from [22, 6, 4). Let $g$ and $\bar{g}$ be geodesically equivalent Riemannian metrics on $M^{n}$. Suppose they are strictly nonproportional at $P \in M^{n}$. Let the function $V$ be compatible with respect to $g$ and $\bar{g}$, and suppose the functions $V_{t}$ satisfy conditions (1).

Then in a small neighborhood $U \subset M^{n}$ of $P$ there exist coordinates (called LeviCivita coordinates) such that the metrics $g, \bar{g}$ and the functions $V_{t}$ are given by the formulas

$$
\begin{aligned}
d s_{g}^{2} & =\sum_{i=1}^{n}(-1)^{i-1} \prod_{j \neq i}\left(\lambda_{j}-\lambda_{i}\right) d x_{i}^{2}, \\
d s_{\bar{g}}^{2} & =\sum_{i=1}^{n} \frac{(-1)^{i-1}}{\lambda_{i} \prod_{\alpha} \lambda_{\alpha}} \prod_{j \neq i}\left(\lambda_{j}-\lambda_{i}\right) d x_{i}^{2}, \\
V_{t} & =\sum_{i=1}^{n}(-1)^{i-1} X_{i} \prod_{j \neq i} \frac{\lambda_{j}-t}{\lambda_{j}-\lambda_{i}},
\end{aligned}
$$


where, for every $i, \lambda_{i}$ and $X_{i}$ are functions of one variable $x_{i}$. If in a neighborhood of almost every point the metrics $g, \bar{g}$ and the functions $V_{t}$ are given by (2)-(4), then the metrics are geodesically equivalent and the functions satisfy conditions (11) with respect to some compatible function $V$.

In the Levi-Civita coordinate system, $L$ is diagonal $\operatorname{Diag}\left(\lambda_{1}, \ldots, \lambda_{n}\right)$. We will always assume that, at every point, the eigenvalues $\lambda_{i}$ of $L$ are indexed according to their value, so that $\lambda_{i}(P) \leq \lambda_{i+1}(P)$ for every $P \in M^{n}$ and every $1 \leq i \leq n-1$.

We see that the metric $g$ and the tensor $L$ define the Levi-Civita coordinate system up to a shift of the origin and a change of the direction of coordinate axes. Indeed, the vector $v_{i} \stackrel{\text { def }}{=} \frac{\partial}{\partial x_{i}}$ is determined up to sign by the conditions

$$
\left\{\begin{array}{cl}
L v_{i} & =\lambda_{i} v_{i} \\
g\left(v_{i}, v_{i}\right) & =(-1)^{i-1} \prod_{j \neq i}\left(\lambda_{j}-\lambda_{i}\right)
\end{array}\right.
$$

In this coordinate system the integrals $\hat{I}_{t}=I_{t}+V_{t}$ (as functions of the cotangent bundle) are given by

$$
\hat{I}_{t}=\sum_{i=1}^{n}(-1)^{i-1}\left(p_{i}^{2}+X_{i}\right) \prod_{j \neq i} \frac{\lambda_{j}-t}{\lambda_{j}-\lambda_{i}} .
$$

Note that the functions $X_{i}$ can be invariantly obtained from $V_{t}$ and $\lambda_{i}$, namely

$$
X_{i}(P)=(-1)^{i-1} V_{\lambda_{i}(P)}(P) .
$$

Let $\hat{I}_{t}^{\prime}=\frac{d}{d t} \hat{I}_{t}$. For every $t$ the function $\hat{I}_{t}^{\prime}$ is a linear combination of the integrals $I_{t_{1}}, \ldots, I_{t_{n}}$.

Corollary 1. Let the metrics $g$ and $\bar{g}$ be geodesically equivalent on a closed connected manifold $M$ and strictly nonproportional at $P \in M$. If a point $(P, \xi) \in T M$ is singular with respect to the Poisson action $\Phi$ corresponding to the integrals $\hat{I}_{t_{i}}=I_{t_{i}}+V_{t_{i}}$, then $d_{(P, \xi)} \hat{I}_{\tilde{\lambda}_{i}}=0$ for some $i$, where $\tilde{\lambda}_{i} \stackrel{\text { def }}{=} \lambda_{i}(P)$. In addition, in Levi-Civita coordinates the $i$-th component of $\xi$ vanishes: $\xi_{i}=0$.

Proof. Consider the function $\hat{I}_{\lambda_{i}(x)}$. Expressed in Levi-Civita coordinates on the cotangent bundle $T^{*} M$, it equals $(-1)^{i-1}\left(p_{i}^{2}+X_{i}\left(x_{i}\right)\right)$, so that its differential is $(-1)^{i-1}\left(2 p_{i} d p_{i}+X_{i}^{\prime}\left(x_{i}\right) d x_{i}\right)$. On the other hand,

$$
d \hat{I}_{\tilde{\lambda}_{i}}=d \hat{I}_{\lambda_{i}\left(x_{i}\right)}-\hat{I}_{\tilde{\lambda}_{i}}^{\prime} d \lambda_{i}\left(x_{i}\right)=(-1)^{i-1} 2 p_{i} d p_{i}+\left((-1)^{i-1} X^{\prime}\left(x_{i}\right)-\lambda_{i}^{\prime}\left(x_{i}\right) \hat{I}_{\tilde{\lambda}_{i}}^{\prime}\right) d x_{i} .
$$

Thus if a linear combination $\sum \mu_{i} d \hat{I}_{\tilde{\lambda}_{i}}$ vanishes, then for every $\mu_{i} \neq 0$ the corresponding $d \hat{I}_{\tilde{\lambda}_{i}}$ vanishes. Then its $d p_{i}$ and $d x_{i}$ components vanish, yielding $p_{i}=0$ (which implies $\xi_{i}=0$ ).

2.4. Submanifolds $\mathcal{M}_{i}$ and $\operatorname{Sing}_{i}$. In this section we assume that $g$ and $\bar{g}$ are geodesically equivalent metrics on a closed connected $M^{n}$, that every eigenvalue $\lambda_{i}$ of $L$ is nonconstant, and that the functions $V_{t}$ satisfy (11) with respect to a compatible $V$. For $i=1, \ldots, n-1$, put

$$
\operatorname{Reg}_{i}=\left\{x \in M: \lambda_{i}(x) \neq \lambda_{i+1}(x)\right\},
$$

and for $i=0$, let $\operatorname{Reg}_{0}=M^{n}$. At every point of $\operatorname{Reg}_{i} \cap \operatorname{Reg}_{i-1}$ the eigenvalue $\lambda_{i}$ is simple. In particular, at every point of $\operatorname{Reg} \stackrel{\text { def }}{=} \bigcap_{i} \operatorname{Reg}_{i}$, the eigenvalues $\lambda_{1}, \ldots, \lambda_{n}$ are mutually distinct. 
For every $x \in \operatorname{Reg}_{i} \cap \operatorname{Reg}_{i-1}$ denote by $\mathcal{D}_{i}(x) \subset T_{x} M^{n}$ the subspace spanned by the eigenspaces corresponding to $\lambda_{j}, j \neq i$. The distribution $\mathcal{D}_{i}$ is smooth. By Theorem 4, it is integrable in $\operatorname{Reg}_{i} \cap \operatorname{Reg}_{i-1}$. Denote by $\mathcal{M}_{i}(P)$ its integral manifold containing $P \in M^{n}$ (beware of our using the notation $D_{\mathrm{C}(i)}$ and $M_{\mathrm{C}(i)}$ in 21] instead of the present $\mathcal{D}_{i}$ and $\left.\mathcal{M}_{i}\right)$. By Theorem 4 the functions $\lambda_{i}$ and $X_{i}$ are constant along $\mathcal{M}_{i}(P)$.

For $i=1, \ldots, n-1$, let $\bar{\lambda}_{i}=\frac{1}{2}\left(\max _{x \in M} \lambda_{i}+\min _{x \in M} \lambda_{i+1}\right)$. By Corollary 1 from 25, for every point $P \in M^{n}$ we have $\lambda_{i}(P) \leq \bar{\lambda}_{i} \leq \lambda_{i+1}(P)$. Consider

$$
\operatorname{Sing}_{i} \stackrel{\text { def }}{=}\left\{P \in M^{n}:\left(\lambda_{i}(P)-\bar{\lambda}_{i}\right)\left(\lambda_{i+1}(P)-\bar{\lambda}_{i}\right)=0\right\} .
$$

In 25] (see Theorem 5 there) it was proven that if $\operatorname{Sing}_{i}$ is nonempty, then, under the assumption that all $\lambda_{i}$ are nonconstant, it is a connected submanifold of codimension 1. Moreover, almost all points of $\mathrm{Sing}_{i}$ belong to Reg and the intersection $\operatorname{Sing}_{i} \cap \operatorname{Reg}_{i}\left(\operatorname{Sing}_{i} \cap \operatorname{Reg}_{i+1}\right.$, respectively) is a finite union of leaves $\mathcal{M}_{i}\left(\mathcal{M}_{i+1}\right.$, respectively).

In 21] (see Lemma 2 there) we proved that if the function $\lambda_{i}$ is not constant, then every $\mathcal{M}_{i}(P)$ is a closed submanifold or is a part of $\operatorname{Sing}_{i}$ or $\operatorname{Sing}_{i+1}$. Combining this observation and Corollary 1, we obtain that the projection of every singular orbit belongs to some compact $\mathcal{M}_{i}$ or to one of $\operatorname{Sing}_{i}$. Since our measure $\mu$ from $\S 2.2$ is ergodic, $\operatorname{Supp}(\mu)$ belongs to the closure of an orbit. Then the projection of $\operatorname{Supp}(\mu)$ belongs to a compact submanifold of smaller dimension.

The last step is to explain that the dynamics on $\operatorname{Supp}(\mu)$ is a subsystem of a certain Benenti system on this submanifold. Almost every point of $\mathrm{Sing}_{i}$ belongs to $\mathcal{M}_{i} \cup \mathcal{M}_{i+1}$, so it is sufficient to consider only $\mathcal{M}_{i}$. Since $\mathcal{M}_{i} \cap \operatorname{Reg}$ is dense in $\mathcal{M}_{i}$, we can use Levi-Civita coordinates. Since $T^{*} \mathcal{M}_{i}$ is a symplectic submanifold of $T^{*} M^{n}$, the claim follows from the fact that the restrictions of the integrals $\hat{I}_{t}$ to $T^{*} \mathcal{M}_{i}$ are linear combinations of the integrals of the induced Benenti system on $\mathcal{M}_{i}$. This assertion can be checked in Levi-Civita coordinates using formula (5).

In fact, the family $V_{t}$ is determined up to a constant polynomial of degree $n-1$. Since the function $X_{i}$ is constant on $\mathcal{M}_{i}(P)$, we can assume without loss of generality that $X_{i}=0$ on $\mathcal{M}_{i}(P)$. Since the coordinate $p_{i}$ vanishes along $T^{*} \mathcal{M}_{i}(P)$ and the function $\lambda_{i}$ is constant on $\mathcal{M}_{i}(P)$, the restriction of the integral $\hat{I}_{t}=I_{t}+V_{t}$ to $T^{*} \mathcal{M}_{i}(P)$ is equal to

$$
\begin{aligned}
\hat{I}_{t}=\sum_{k \neq i}(-1)^{k-1}\left(p_{k}^{2}+X_{k}\right) \prod_{j \neq k} \frac{\lambda_{j}-t}{\lambda_{j}-\lambda_{k}} \\
=\left(\lambda_{i}-t\right) \cdot\left[\sum_{k \neq i} \frac{(-1)^{k-1-\theta(k-i)}\left(p_{k}^{2}+X_{k}\right)}{\left|\lambda_{i}-\lambda_{k}\right|} \prod_{j \neq k, i} \frac{\lambda_{j}-t}{\lambda_{j}-\lambda_{k}}\right],
\end{aligned}
$$

where $\theta(x)$ is the Heaviside function, so that $\psi(k)=k-\theta(k-i)$ enumerates $\{1, \ldots, n\} \backslash\{i\}$.

By the direct calculation we check that, for every $t$, the above expression in square brackets is a linear combinations of the integrals $\hat{I}_{\tau_{1}}^{\text {new }}, \ldots, \hat{I}_{\tau_{n-1}}^{\text {new }}$ of the Benenti system corresponding to geodesically equivalent metrics $g^{\text {new }} \stackrel{\text { def }}{=} g_{\left.\right|_{\mathcal{M}_{i}(P)}}, \bar{g}^{\text {new }} \stackrel{\text { def }}{=}$ $\lambda_{i} \bar{g}_{\mid \mathcal{M}_{i}(P)}$ on $\mathcal{M}_{i}(P)$ and the family $V_{t}^{\text {new }}: \mathcal{M}_{i}(P) \rightarrow \mathbb{R}, V_{t}^{\text {new }} \stackrel{\text { def }}{=} \frac{1}{\lambda_{i}-t} V_{t \mid \mathcal{M}_{i}(P)}$. The facts that the metrics $g^{\text {new }}, \bar{g}^{\text {new }}$ are geodesically equivalent and strictly nonproportional at least at one point and that the family $V_{t}$ satisfies condition (11) 
follow from Theorem 4 , because on $\mathcal{M}_{i}(P)$ the coefficient $\left|\lambda_{k}-\lambda_{i}\right|$ depends on $x_{k}$ only and therefore can be "hidden" in the corresponding $d x_{k}$ and $X_{k}$. Thus our system is a subsystem of a Benenti system and the induction hypothesis finishes the proof of Theorem 1 .

\section{Discussion}

It is clear that all the difficulties with vanishing of entropies for integrable systems are due to a complicated singularity set (we explained essentially in $\$ 2.2$ that the set of regular points bears no entropy), as positive-entropy examples of [7, 20, 9, 10] demonstrate. In all good cases, where vanishing of the entropy has been proven, some stratification of singularities was achieved; see, e.g., 28, 29, 33, 9, 21]. Here we formulate a scheme for most vanishing results.

We consider systems on noncompact $W^{2 n}$, but such that the variational principle holds. This is, for instance, the case when $W^{2 n}$ admits an exhaustion by compact invariant sets (other cases are discussed in [32]). Define the following $\Phi$-invariant subsets of $W^{2 n}$ :

$$
\Sigma_{k}=\left\{x \in W^{2 n} \mid \operatorname{rank}\left(d_{x} \Phi\right)=k\right\} .
$$

Theorem 5. Suppose for every $k<n$ we can decompose $\Sigma_{k}=\Sigma_{k}^{+} \cup \Sigma_{k}^{-}$, where $\Sigma_{k}^{+}$ is a closed invariant subset of $\Sigma_{k}$ and $\Sigma_{k}^{-}$consists of nonrecurrent points of $\Phi(v)$ for almost every $v \in \mathbb{R}^{n}$. Suppose the momentum map $\Psi: \Sigma_{k}^{+} \rightarrow \mathbb{R}^{n}$ can be factorized to the composition of continuous maps $\pi_{k}: \Sigma_{k}^{+} \rightarrow A_{k}$ to a Hausdorff space $A_{k}$ and $\sigma_{k}: A_{k} \rightarrow \mathbb{R}^{n}$ such that each fiber $\Sigma_{k}^{\alpha}=\pi_{k}^{-1}(\alpha)$ is a $\Phi$-invariant $k$-dimensional submanifold of $W^{2 n}$. Then the entropy pseudonorm vanishes: $\rho_{\Phi} \equiv 0$.

Proof. By the variational principle it suffices to prove $h_{\mu}(\Phi(v))=0$ for almost every $v$ and every $\Phi(v)$-invariant ergodic measure $\mu$. By ergodicity $\Psi$ is constant on the support of $\mu$. Consequently, it suffices to prove that for every $k$ the system has zero entropy on $\Psi^{-1}(c) \cap \Sigma_{k}$. Since $\mathcal{R}(v) \cap \Sigma_{k} \subset \Sigma_{k}^{+}$, where $\mathcal{R}(v)$ denotes the set of $\Phi(v)$-recurrent points, it is enough to show vanishing of entropy on the set $\Sigma_{k}^{+} \cap \Psi^{-1}(c)$.

This set is foliated by the strata $\Sigma_{k}^{\alpha}$, and hence $\mu$ must be supported on a single connected component of it only. This component possesses a transitive Poisson $\mathbb{R}^{k}$-action and so is isomorphic to a torus $T^{k}$ (it cannot be a cylinder $T^{k-l} \times \mathbb{R}^{l}$ because it consists of recurrent points) with quasi-periodic dynamics and hence $h_{\mu}\left(\Phi(v) \mid \Sigma_{k}^{+} \cap \Psi^{-1}(c)\right)=0$ implying the claim.

The assumptions of Theorem 5 are satisfied for integrable systems with Williamson-Vey-Eliasson-Ito nondegenerate singularities (see [12, 18]) or with Taimanov nondegeneracy condition [33].

Indeed, Taimanov's condition is as follows: for every $(n-k) \times n$ matrix $v_{i j}$ of rank $n-k$ the set

$$
\left\{\xi \in \Sigma_{k}: \xi \neq 0 \text { and } \sum_{j=1}^{n} v_{i j} d_{\xi}\left(I_{j}\right)=0 \quad \forall i=1, \ldots, n-k\right\}
$$

is a submanifolds of $T M$ of dimension k. Clearly, if the Taimanov condition is fulfilled, the space of the connected components of the sets

$$
L_{k}(c) \stackrel{\text { def }}{=} \Sigma_{k} \cap \Psi^{-1}(c)=\left\{\xi \in \Sigma_{k}: I_{1}=c_{1}, \ldots, I_{n}=c_{n}\right\}
$$


with induced topology is a Hausdorff space, so that the assumptions of Theorem 5 are automatically fulfilled.

The definition of Williamson-Vey-Eliasson-Ito nondegenerate singularities is complicated and can be found, for example, in Section 1.8 of the book [5]. It is a generalization of the notion "Morse singularity" for integrable systems. Locally, the structure of the momentum mapping near Williamson-Vey-Eliasson-Ito nondegenerate points is given by Eliasson-Ito theorem, which is a generalization of the Morse lemma to the context of integrable Hamiltonian systems (Section 1.8.3 of [5]). From this description, it is clear that the space of connected components of the sets $L_{k}(c)$ given by (7) with induced topology is again a Hausdorff space, so that the assumptions of Theorem 5 are fulfilled.

Our induction approach implies that the singularities of Benenti systems are also stratified in the manner of Theorem [5. It is feasible that a kind of good stratification is necessary for vanishing of the entropies.

Acknowledgments. We thank Professors Butler, Paternain, Pesin, and Taimanov for useful discussions. The second author thanks the University of Troms $\varnothing$, where the essential part of the results were obtained, for hospitality, and DFG-programm 1154 (Global Differential Geometry) and Ministerium für Wissenschaft, Forschung und Kunst Baden-Württemberg (Eliteförderprogramm Postdocs 2003) for partial financial support. The first author expresses his gratitude to Stanford University and Penn State University, where he was a visitor while working on this paper.

\section{REFERENCES}

[1] V.I. Arnold, Mathematical methods of classical mechanics, Nauka, Moscow; English transl., Graduate Texts in Mathematics, Springer, 1989. MR.1037020 (93c:70001)

[2] S. Benenti, Inertia tensors and Stäckel systems in the Euclidean spaces, Differential geometry (Turin, 1992). Rend. Sem. Mat. Univ. Politec. Torino 50 (1993), no. 4, 315-341. MR:1261446 (95c:70020)

[3] S. Benenti, An outline of the geometrical theory of the separation of variables in the Hamilton-Jacobi and Schrödinger equations, SPT 2002: Symmetry and perturbation theory (Cala Gonone), 10-17, World Sci. Publishing, River Edge, NJ, 2002. MR1976651 (2004a:70022)

[4] S. Benenti, Special Symmetric Two-tensors, Equivalent Dynamical Systems, Cofactor and Bi-Cofactor Systems, Acta Applicandae Mathematicae 87 (2005), no. 1-3, 33-91. MR2151124

[5] A. V. Bolsinov, A. T. Fomenko, Integrable Hamiltonian systems. Geometry, topology, classification, Chapman \& Hall, 2004. MR2036760 (2004j:37106)

[6] A.V. Bolsinov, V.S. Matveev, Geometrical interpretation of Benenti's systems, Journ. Geom. Phys. 44 (2003), 489-506. MR1943174 (2005d:37117)

[7] A. V. Bolsinov, I. A. Taimanov, Integrable geodesic flows with positive topological entropy, Invent. Math. 140 (2000), 639-650. MR1760753 (2001b:37081)

[8] R. Bowen, Entropy for group endomorphisms and homogeneous spaces, Trans. A.M.S. 153 (1971), 401-414. MR0274707 (43:469)

[9] L. T. Butler, G. P. Paternain, Collective geodesic flows, Ann. Inst. Fourier (Grenoble) 53 (2003) no. 1, 265-308. MR.1973073 (2004m:37054)

[10] L. T. Butler, Toda lattices and positive-entropy integrable systems, Invent. Math. 158 (2004), no. 3, 515-549. MR2104793 (2005h:37122)

[11] M. Crampin, W. Sarlet, G. Thompson, Bi-differential calculi, bi-Hamiltonian systems and conformal Killing tensors, J. Phys. A 33 (2000), no. 48, 8755-8770. MR1801467 (2001k:37094)

[12] L. H. Eliasson, Normal forms for Hamiltonian systems with Poisson commuting integrals. Elliptic case, Comment. Math. Helv. 65 (1990), no. 1, 4-35. MR.1036125 (91d:58223) 
[13] J. P. Conze, Entropie d'un groupe abelien de transformations, Z. Wahrsch. 25 (1972), 1130. MR 0335754 (49:534)

[14] V. Guillemin, S. Sternberg, On collective complete integrability according to the method of Thimm, Ergod. Th. \& Dynam. Sys. 3 (1983), no. 2, 219-230. MR0742224 (85i:58057)

[15] K. H. Hofmann, L. N. Stojanov, Topological entropy of group and semigroup actions, Adv. Math. 115 (1995), no. 1, 54-98. MR.1351326 (97a:22006)

[16] Y. Hu, Some ergodic properties of commuting diffeomorphisms, Ergod. Th. \& Dynam. Sys. 13 (1993), 73-100. MR 1213080 (94b:58061)

[17] A. Ibort, F. Magri, G. Marmo, Bihamiltonian structures and Stäckel separability, J. Geom. Phys. 33 (2000), no. 3-4, 210-228. MR 1747040 (2001a:37082)

[18] H. Ito, Action-angle coordinates at singularities for analytic integrable systems, Math. Z. 206 (1991), 363-407. MR1095762 (92e:58069)

[19] A. Katok, B. Hasselblatt, Introduction to the modern theory of dynamical systems, Encyclopedia of Math. and its Appl. 54, Cambridge University Press, Cambridge, 1995. MR:1326374 (96c:58055)

[20] B. Kruglikov, Examples of integrable sub-Riemannian geodesic flows, Jour. Dynam. Contr. Syst. 8 (2002), no. 3, 323-340. MR1914446 (2003m:37081)

[21] B. S. Kruglikov, V. S. Matveev, Strictly nonproportional geodesically equivalent metrics have $h_{\mathrm{top}}(g)=0$, Ergod. Th. \& Dynam. Sys. 26 (2006), 219-245.

[22] T. Levi-Civita, Sulle trasformazioni delle equazioni dinamiche, Ann. di Mat., serie $2^{a}, \mathbf{2 4}$ (1896), 255-300.

[23] H. Lundmark, S. Rauch-Wojciechowski, Driven Newton equations and separable timedependent potentials, J. Math. Phys. 43 (2002), no. 12, 6166-6194. MR1939638 (2004j:70035)

[24] V. Matveev, P. Topalov, Trajectory equivalence and corresponding integrals, Regular and Chaotic Dynamics 3 (1998), no. 2, 30-45. MR1693470 (2000d:37068)

[25] V.S. Matveev, Hyperbolic manifolds are geodesically rigid, Invent. Math. 151 (2003), 579609. MR1961339 (2004f:53044)

[26] V.S. Matveev, Three-dimensional manifolds having metrics with the same geodesics, Topology 42 (2003), no. 6, 1371-1395. MR1981360 (2004g:53095)

[27] V.S. Matveev, Projectively equivalent metrics on the torus, Diff. Geom. Appl. 20 (2004), 251-265. MR2053913 (2005c:53108)

[28] G. Paternain, On the topology of manifolds with completely integrable geodesic flows. I, Ergod. Th. \& Dynam. Sys. 12 (1992), 109-121. MR1162403 (93g:58117)

[29] G. Paternain, On the topology of manifolds with completely integrable geodesic flows. II, Journ. Geom. Phys. 123 (1994), 289-298. MR1269245 (95b:58115)

[30] G. Paternain, Geodesic flows, Birkhäuser, 1999. MR.1712465 (2000h:53108)

[31] G. P. Paternain, J. Petean, Zero entropy and bounded topology, preprint: ArXiv.org/math.DG/0406051; to appear in Comment. Math. Helv.

[32] Y. Pesin, Dimension theory in dynamical systems, Chicago Lect. in Math. Ser., The University of Chicago Press, 1997. MR 1489237 (99b:58003)

[33] I. A. Taimanov, Topology of Riemannian manifolds with integrable geodesic flows, Proc. Steklov Inst. Math. 205 (1995), 139-150. MR1428676 (98b:58135)

Institute of Mathematics and Statistics, University of Troms $\varnothing$, Troms $\varnothing$ 90-37, NorWAY

E-mail address: kruglikov@math.uit.no

Mathematisches Institut der Albert-Ludwigs-Universität, Eckerstrasse-1, Freiburg 79104, GERMANY

E-mail address: matveev@email.mathematik.uni-freiburg.de 